\title{
Role of kallistatin in pediatric patients with pulmonary arterial hypertension
}

\author{
Özge Pamukçu ${ }^{1 \oplus}$, Derya Ay ${ }^{1 \oplus}$, Kazım Üzüm $^{1 \oplus}$, Didem Barlakketi² ${ }^{2 \oplus}$, Mehmet Köse ${ }^{3 \oplus}$, \\ Ali Baykan ${ }^{1 \oplus}$, Süleyman Sunkak ${ }^{1 \oplus}$, Nazmi Narin ${ }^{1 \oplus}$ \\ Divisions of ${ }^{1}$ Pediatric Cardiology, and ${ }^{3}$ Pediatric Pulmonology, Department of Pediatrics, ${ }^{2}$ Department of Biochemistry, Erciyes \\ University Faculty of Medicine, Kayseri, Turkey.
}

\begin{abstract}
Background and objectives. Kallistatin, a serine proteinase inhibitor, exerts its effect by vascular repair, angiogenesis inhibition, strong vasodilation, inhibition of vascular endothelial growth factor (VEGF), antiinflammation, and anti-apoptosis. We hypothesized as to whether it has a protective role in pulmonary arterial hypertension $(\mathrm{PAH})$.

Methods. The study included 5 subgroups (78 patients; 44 male): Eisenmenger syndrome ( $\mathrm{n}=16)$, PAH with left to right shunt $(n=20)$, idiopathic PAH $(n=7)$, patients with left to right shunt without PAH $(n=19)$, and patients with innocent heart murmur $(\mathrm{n}=16)$. Physical examination, chest radiography, electrocardiography, and transthoracic echocardiography (TTE) were performed for each patient. PAH diagnosis was confirmed by catheterization. Serum kallistatin, tumor necrosis factor alpha (TNF- $\alpha$ ), Interleukin-10 (IL-10) and N-terminal pro b-type natriuretic peptide (NT-proBNP) levels were studied for each patient.

Results. The lowest median kallistatin value was found in Eisenmenger syndrome: 1.19 (0.87-3.30) $\mu \mathrm{g} / \mathrm{ml}$. The highest value belonged to control group with innocent murmur: 2.89 (1.19-5.66) $\mu \mathrm{g} / \mathrm{ml}$. Serum levels of kallistatin were significantly lower in patients with PAH $(\mathrm{p}<0.05)$. TNF- $\alpha$ values were increased and IL-10 values were decreased in pulmonary hypertension. However; no correlation was found between kallistatin levels and cytokines.

Conclusions. Kallistatin may have a protective effect in pulmonary arterial hypertension by repairing vascular damage, inhibition of angiogenesis, strong vasodilator effect, inhibiting VEGF, and anti-inflammatory mechanism of action. To our knowledge, our study is the first one that shows the role of kallistatin in pulmonary hypertension. Kallistatin may represent a promising novel therapeutic approach for pulmonary hypertension in the near future.
\end{abstract}

Key words: kallistatin, pulmonary hypertension, TNF $\alpha$, IL-10.

Pulmonary hypertension is a progressive obliterative vasculopathy. Underlying mechanisms are very complex; there is not a single but group of events. The one that takes the main role seems to be endothelial dysfunction. Other events are changes in

○ Özge Pamukçu

ozgepamukcu2002@yahoo.com

Received 7th December 2018, revised 5th March 2019, accepted 16th April 2019.

The study was presented as oral presentation at the $16^{\text {th }}$ National Pediatric Cardiology and Cardiac Surgery Congress, 19-22 April 2017, Antalya, Turkey. function of platelet and ion channels, calcium homeostasis, inflammation, angiogenesis etc. ${ }^{1}$ Pulmonary arterial hypertension (PAH) is a subgroup of pulmonary hypertension that has idiopathic, genetic, and comorbid etiologies (e.g., connective tissue disorders, HIV infection, schistosomiasis, etc.). ${ }^{2}$ It is characterized by occlusion of pulmonary arterioles that results the increase in the pulmonary vascular resistance, right ventricular hypertrophy, and finally right sided heart failure.

In recent years, there is an increased understanding of the pathophysiology of 
pulmonary hypertension which is thought to be helpful in finding the novel PAH-specific therapies.

Kallistatin is a serine proteinase inhibitor originally known as a tissue kallikrein inhibitor. The main ways of action of it are inhibiting inflammation, oxidative stress, apoptosis, and angiogenesis. ${ }^{3,4}$ Herein; by this study, we aimed to discuss the protective role of kallistatin in pulmonary arterial hypertension. Perhaps in near future it may represent a promising novel therapeutic approach for pulmonary hypertension.

\section{Material and Methods}

The study was performed in our university between December 2015- January 2017. The study was approved by the Institutional Ethics Committee (06.02.2015; 2015/799).

The study included a total of 78 patients. The study population was classified under 5 groups. Group 1: Eisenmenger syndrome, Group 2: $\mathrm{PAH}$ patients with left to right shunt, Group 3: idiopathic PAH, Group 4: patients with left to right shunt but without $\mathrm{PAH}$, Group 5 (controls): patients with innocent murmur.

The following data for each patient were obtained: age, weight, sex, and non-cardiac medical problems. Underlying cardiac pathology was recorded except for groups 3 and 5. Physical examination, chest radiography, electrocardiography, and transthoracic echocardiography (TTE) were done for each patient. Parents were informed about the study and written consent was taken from each of them before being included in the study. In addition, pulmonary hypertensive ones (Group $1,2,3)$ were also informed about cardiac catheterization and its complications; a written consent was also taken for the procedure from the parents.

\section{Cardiac catheterization}

Angiography was performed in pulmonary hypertensive patients. Following heparinization and a dose of intravenous antibiotic, hemodynamic and angiographic evaluations were performed. The procedure included measurements of pressure (systolic and diastolic and/or mean) and oxygen saturation in the superior vena cava, inferior vena cava, right atrium, pulmonary artery, right ventricle $(\mathrm{RV})$, and the aorta. Pressure recordings were obtained by using fluid-filled catheter systems, and these systems were calibrated to zero at the midaxillary line.

The diagnosis of each patient was determined according to current guidelines ${ }^{5}$ (mean pulmonary artery pressure-PAP $\geq 25 \mathrm{~mm} \mathrm{Hg}$, pulmonary artery wedge pressure $<15 \mathrm{~mm} \mathrm{Hg}$ ).

According to the clinical classification by Simonneau et al. ${ }^{6}$; Eisenmenger's syndrome includes all intra- and extra-cardiac defects as well as systemic-to-pulmonary shunts.

Idiopathic PAH is defined in the guidelines as PAH with no underlying disease known to be associated.

\section{Serum kallistatin, cytokine, and NT-proBNP levels}

Blood samples for kallistatin was taken by the tubes centrifuged at $4000 \mathrm{rpm}$ for $10 \mathrm{~min}$ at 4 $1 \mathrm{C}$. The serum was kept at $-80^{\circ} \mathrm{C}$ as frozen until required. Kallistatin levels were determined by ELISA (R\&D Systems, Inc. Minneapolis, USA) as previously described. ${ }^{7}$

Concentrations of TNF- $\alpha$ and IL-10 in plasma were measured in picograms per milliliter (pg/ $\mathrm{ml}$ ) by commercially available enzyme-linked immunosorbent assay kits (Cayman Chemical, Ann Arbor, MI, USA).

Five $\mathrm{ml}$ blood samples were drawn from both patient and control groups for $\mathrm{N}$ terminal pro brain natriuretic peptide (NT-proBNP). Blood samples were centrifuged at $+4^{\circ} \mathrm{C}$ and 1500 rpm for 5 minutes. Plasma part of the upper phase was taken in to another tube for NTproBNP calculation. Samples were preserved at $-80^{\circ} \mathrm{C}$ till the study date. Later on, they were 
studied with ELISA method using Biomedica NT-proBNP N-terminal pro-BNP commercial kits (NT- ProBNP enzyme immuno assay kit Biomedica, Bratislava, Slovakia) and Elecsys ${ }^{\circledR}$ 1010 aoutoanalyzer (Roche Diagnostics, Basel, Switzerland). Results were expresses as fmol/ $\mathrm{ml}(1 \mathrm{fmol} / \mathrm{ml}=16.1 \mathrm{pg} / \mathrm{ml})$.

\section{Statistical analysis}

The Shapiro-Wilk test was used to analyze the distribution of the data. If the data presented with a normal distribution, analysis of variance (ANOVA) followed by the Tukey's test was used to evaluate the results. Comparisons of means were performed with Student's t-test. Comparisons of medians were performed with the Mann-Whitney U-test. Correlations were calculated with Pearson product moment or Spearman rank order, as determined by the normalcy of data distribution. Differences were considered significant at $p<0.05$.

\section{Results}

Totally 78 patients [44 male with a mean age of $12.1 \pm 4.3$ (range: $3-18$ years), 34 female with a mean age of $12.0 \pm 4.1$ (range: $4-17$ years)] were included in the study. The first group had 16 patients with Eisenmenger syndrome. Underlying congenital heart diseases of this group were ventricular septal defect $(n=$ 13) and patent ductus arterious $(n=13)$. The second group had 20 pulmonary hypertensive patients with left to right shunt. Group 3 included 7 patients with idiopathic pulmonary hypertension. Group 4 had 19 patients with left to right shunt but without pulmonary arterial hypertension. The last group, Group 5 included the 16 patients with innocent heart murmur.

In Group 1, 6 patients used endothelin receptor antagonist (bosentan/macitentan) treatment as a monothereapy. The rest of the patients in group 1 had combination therapy (bosentan/ macitentan+ phosphodiesterase-5 inhibitor sildenafil, bosentan/macitentan+ PGI2 analog (ilioprost), bosentan/macitentan+ ilioprost+ sildenafil). Bosentan was switched to macitentan due to drug side effects in 5 patients. In group 3 , vasoreactivity was negative in all patients. Two patients received only endothelin receptor antagonist (macitentan). The rest of the 5 patients received combination therapy (endothelin receptor antagonist + sildenafil or endothelin receptor antagonist+ sildenafil+ilioprost).

Median serum kallistatin levels were summarized for each group in Table I. The lowest value was found in Group 1 (Eisenmenger syndrome); the highest value belonged to control group with innocent murmur. Serum levels of kallistatin were significantly lower $(p<0.05)$ in pulmonary hypertensive patients (Group 1, 2, 3). However; no statistically significant difference was detected between those 3 groups. Negative correlation was detected between mean pulmonary arterial pressure and serum kallistatin levels (rho: - 0.303; $\mathrm{p}=0.009$ ).

Mean value of TNF- $\alpha$ of pulmonary hypertensive group was $10.5 \pm 4.3 \mathrm{pg} / \mathrm{ml}$, TNF- $\alpha$ of control group was $9.7 \pm 2.4 \mathrm{pg} / \mathrm{ml}$. There was no statistically significant difference between these two groups. Mean value of IL-10 of pulmonary hypertension group was $14.6 \pm 2.82 \mathrm{pg} / \mathrm{ml}$ and

Table I. Median serum kallistatin and NT-proBNP levels according to groups.

\begin{tabular}{|c|c|c|}
\hline Groups & Kallistatin $(\mu \mathrm{g} / \mathrm{ml})^{*}$ & NT ProBNP levels $(\mathrm{pg} / \mathrm{ml})^{*}$ \\
\hline Group 1: Eisenmenger syndrome $(n=16)$ & $1.19(0.87-3.30)$ & $123(20-3,586)$ \\
\hline Group 2: Left to right shunt with PAH (n=20) & $1.79(1.13-3.60)$ & $657(20-4,840)$ \\
\hline Group 3: Idiopathic PAH (n=7) & $1.51(1.07-2.02)$ & $510(125-2,470)$ \\
\hline Group 4: Left to right shunt without PAH $(n=19)$ & $2.52(0.87-3.21)$ & $57(20-185)$ \\
\hline Group 5: Control patients $(n=16)$ & $2.89(1.19-5.66)$ & $20(20-51)$ \\
\hline
\end{tabular}

${ }^{*}$ Data are presented as median (minimum - maximum) PAH: pulmonary arterial hypertension. 
control group was $15 \pm 4 \mathrm{pg} / \mathrm{ml}$. In addition; no correlation was found between kallistatin levels and cytokines TNF- $\alpha$ and IL-10.

Median NT-proBNP levels for groups are summarized in Table I. There was a statistically significant difference between the Eisenmenger group and the control group $(\mathrm{p}<0.05)$. However; there was no significant differences between pulmonary hypertensive groups such as Groups 1,2 , and 3 . Moreover, there was no statistically significant difference between the control group and the left to right shunt group without PAH. NT-proBNP levels of pulmonary hypertensive groups were significantly higher than the patients without pulmonary hypertension ( $p$ $<0.05)$.

Bosentan treatment was started in 39 patients $(92.8 \%)$, but in $10(23.8 \%)$ patients, bosentan was switched to macitentan due to drug side effects or shortening in 6 minutes walking distance. Bosentan, sildenafil and iloprost combined therapy was started in one of the patients for poor clinical status. In one patient sildenafil and in another patient single macitentan treatment was started. In follow up, 29 patients (69\%) received only bosentan. Of the 42 patients, $9(21.4 \%)$ received combined drug therapy consisting of endothelin receptor blocker, phosphodiesterase-5 inhibitor or PGI2 analog. In patients who received bosentan, liver toxicity was seen in 1 patient and thrombocytopenia in 4 patients. Macitentan was used in 12 patients aged between 14 and 22 years who received single or combined treatment. The duration of macitentan use was 16.5 months in these patients.

\section{Discussion}

Pulmonary arterial hypertension is a big burden for the healthcare system. Morbidity is high and prognosis is poor. There is no ideal treatment that can reverse or prevent the disease process. Treatment given for symptomatic relief is long term and expensive which means a huge financial load. Also most pulmonary hypertensive patients should have sedentary life that means the loss of manpower. Further understanding the pathogenesis may lead us to take steps in the development of new treatment strategies. If the quality of life of such patients improves, they may participate in work life more effectively and contribute to country economics.

Kallistatin is an endogenous protein; a member of the serine proteinase inhibitors. In previous studies it was shown that kallistatin has a role in vascular repair, angiogenesis inhibition, strong vasodilatation, VEGF inhibition, antiinflammation and anti-apoptosis. The most conspicuous point is that all of these events have a role in the pathogenesis of pulmonary hypertension and we hypothesized that kallistatin may have a protective role in pulmonary hypertension. In order to support this hypothesis, we could not find any study in literature concerning the role of kallistatin in pulmonary hypertension. Therefore, we discuss other studies about kallistatin that might be a clue for preventing pulmonary hypertension. The main event in the pathogenesis of pulmonary arterial hypertension is endothelial dysfunction and it has been reported that kallistatin had a role in vascular repair by promoting mobility, viability, and function of endothelial progenitor cells. ${ }^{8,9}$

Vascular smooth muscle proliferation is also known to be an important mechanism in pulmonary hypertension and kallistatin was found to have role in the inhibition of angiogenesis in hepatocellular carcinoma ${ }^{10}$, which also promotes apoptosis and autophagy of breast cancer cell. ${ }^{11}$ The other mechanism of pulmonary hypertension is increased intravascular thrombosis and vasodilator effect of kallistatin was proven previously by Chao et al. ${ }^{12}$ Vascular endothelial growth factor is known to be increased in pulmonary arterial hypertension. In breast and hepatocellular cancer kallistatin was demonstrated to inhibit VEGF secretion. ${ }^{13}$ 
Inflammation is a major feature of pulmonary arterial hypertension. Immune processes are altered; there is usually a failure to resolve inflammation. As a result of inflammation circulating levels of certain cytokines and chemokines are abnormally elevated that lead to vascular remodeling in pulmonary arterial hypertension.

In our study we investigated the role of TNF- $\alpha$ and IL-10 in pulmonary arterial hypertension and its relation with kallistatin. We have found that TNF- $\alpha$ increased and IL-10 level decreased in our study; but there was no statistically significant difference which could be related to the small number of patients. Decrement in IL-10 level could be explained as consumption of anti-inflammatory cytokines during the inflammatory process (pulmonary hypertension). In fact, kallistatin levels were found to be significantly low in pulmonary hypertension; no correlation was found between cytokine and kallistatin levels.

Soon et al. ${ }^{14}$ described that TNF- $\alpha$ and IL-10 levels were significantly raised in the $\mathrm{PAH}$ group as a whole compared with healthy control subjects. They suggested that increased levels of anti-inflammatory cytokines such as IL-10 might be related to compensatory mechanisms also another important detail was high IL-10 found in patients who were prescribed prostanoids.

The anti-inflammatory role of kallistatin was studied in various organ systems like kidney, liver, heart and lungs. Its protective effects were shown in salt-induced renal injury, inflammation, and fibrosis; carbon tetrachlorideinduced liver fibrosis; and cardiac remodeling after chronic myocardial infarction. ${ }^{15-18}$ Furthermore, arthritis in a rat model was shown to be inhibited by adenovirus-mediated human kallistatin gene therapy. ${ }^{19}$

Reduced kallistatin levels are strongly associated with increased lung inflammation and mortality of sepsis-related ARDS in hospitalized patients by Lin et al. ${ }^{20}$. They came to the conclusion that kallistatin ameliorates lipopolysaccharide- induced lung injury and inflammation which could be supportive data for our hypothesis.

Consequently, kallistatin may have a protective effect in pulmonary arterial hypertension by repairing vascular damage, inhibition of angiogenesis, strong vasodilator effect, inhibiting VEGF, anti-inflammatory mechanism of action.

The main restriction of the study is that our study is the only one in the literature therefore we do not have a chance to compare it with others. The number of patients is limited. Low levels of kallistatin may be related to either a decreased production or increase in consumption.

It would be better if we had the chance to compare kallistatin levels of patients before and after starting anti-pulmonary hypertensive drugs to evaluate the effectiveness of the therapy.

\section{REFERENCES}

1. Galie N, Simonneau G. The fifth world symposium on pulmonary hypertension. J Am Coll Cardiol 2013; 62 (Suppl 25): D1-D3.

2. Archer SL, Weir EK, Wilkins MR. Basic science of pulmonary arterial hypertension for clinicians: new concepts and experimental therapies. Circulation 2010; 121: 2045-2066.

3. Chao J, Schmaier A, Chen LM, Yang Z, Chao L. Kallistatin, a novel human tissue kallikrein inhibitor: Levels in body fluids, blood cells, and tissues in health and disease. J Lab Clin Med 1996; 127: 612620 .

4. Miao RQ, Agata JA, Chao L. Chao J. Kallistatin is a new inhibitor of angiogenesis and tumor growth. Blood 2002; 100: 3245-3252.

5. Apitz C, Hansmann G, Schranz D. Hemodynamic assessment and acute pulmonary vasoreactivity testing in the evaluation of children with pulmonary vascular disease. Expert consensus statement on the diagnosis and treatment of paediatric pulmonary hypertension. The European Paediatric Pulmonary Vascular Disease Network, endorsed by ISHLT and DGPK. Heart 2016; 102(Suppl 2): 23-29.

6. Simonneau G, Gatzoulis MA, Adatia I, et al. Updated clinical classification of pulmonary hypertension. J Am Coll Cardiol 2013; 24(Suppl 25): D34-D41. 
7. Wolf WC, Harley RA, Sluce D, Chao L, Chao J. Cellular localization of kallistatin and tissue kallikrein in human pancreas and salivary glands. Histochem Cell Biol 1998; 110: 477-484.

8. Gao L, Li P, Zhang J, et al. Novel role of kallistatin in vascular repair by promoting mobility, viability, and function of endothelial progenitor cells. J Am Heart Assoc 2014; 3: e001194.

9. Liu X, McBride J, Zhou Y, Liu Z, Ma JX. Regulation of endothelial progenitor cell release by Wnt signaling in bone marrow. Invest Ophthalmol Vis Sci 2013; 54: 7386-7394.

10. Tse LY, Sun X, Jiang H, et al. Adeno-associated virusmediated expression of kallistatin suppresses local and remote hepatocellular carcinomas. J Gene Med 2008; 10: 508-517.

11. Li P, Guo Y, Bledsoe G, Yang Z, Chao L, Chao J. Kallistatin induces breast cancer cell apoptosis and autophagy by modulating Wnt signaling and microRNA synthesis. Exp Cell Res 2016; 340: 305314.

12. Chao J, Stallone JN, Liang YM, Chen LM, Wang DZ, Chao L. Kallistatin is a potent new vasodilator. J Clin Invest 1997; 100: 11-17.

13. Jiang $X$, Li $H$, Qiao $H$, Jiang $H, X u R$, Sun $X$. Combining kallistatin gene therapy and meloxicam to treat hepatocellular carcinoma in mice. Cancer Sci 2009; 100: 2226-2233.

14. Soon E, Holmes AM, Treacy CM, et al. Elevated levels of inflammatory cytokines predict survival in idiopathic and familial pulmonary arterial hypertension. Circulation 2010; 122: 920-927.
15. Gao L, Yin H, S Smith R Jr, Chao L, Chao J. Role of kallistatin in prevention of cardiac remodeling after chronic myocardial infarction. Lab Invest 2008; 88: 1157-1166.

16. Shen B, Hagiwara M, Yao YY, Chao L, Chao J Salutary effect of kallistatin in salt-induced renal injury, inflammation, and fibrosis via antioxidative stress. Hypertension 2008; 51: 1358-1365.

17. Diao Y, Zhao XF, Lin JS, Wang QZ, Xu RA. Protection of the liver against $\mathrm{CCl} 4$-induced injury by intramuscular electrotransfer of a kallistatinencoding plasmid. World J Gastroenterol 2011; 17: 111-117.

18. Liu Y, Bledsoe G, Hagiwara M, Shen B, Chao L, Chao J. Depletion of endogenous kallistatin exacerbates renal and cardiovascular oxidative stress, inflammation, and organ remodeling. Am J Physiol Renal Physiol 2012; 303: F1230-F1238.

19. Hsieh JL, Shen PC, Shiau AL, et al. Adenovirusmediated kallistatin gene transfer ameliorates disease progression in a rat model of osteoarthritis induced by anterior cruciate ligament transection. Hum Gene Ther 2009; 20: 147-158.

20. Lin WC, Chen CW, Huang YW, et al. Kallistatin protects against sepsis-related acute lung injury via inhibiting inflammation and apoptosis. Sci Rep 2015; 5: 12463. 